\title{
Propiedades Psicométricas de una Forma Breve del Cuestionario de Parentalidad Alabama en Familias de Preescolares Chilenos
}

\author{
Psychometric Properties of a Brief Form of the Alabama Parenting Questionnaire in \\ Chilean Preschoolers' Families
}

\author{
Félix Cova ${ }^{1}$, Claudio Bustos ${ }^{2}$, Paulina Rincón ${ }^{3}$, Pamela Grandón ${ }^{4}$, Sandra Saldivia ${ }^{5}$ y Carolina \\ Inostroza $^{6}$
}

\begin{abstract}
Resumen
El Cuestionario de Parentalidad de Alabama (CAP) es un instrumento de amplia difusión para evaluar prácticas parentales. Estudios recientes muestran positivas cualidades psicométricas y una estructura factorial robusta de una forma breve de él. El objetivo de este estudio fue evaluar las propiedades psicométricas de una forma breve del CAP al usarse con figuras parentales de niños y niñas de dos a seis años en Chile. Los participantes fueron figuras parentales, madres principalmente, de una muestra de 558 niños y niñas de 2 a 6 años. El análisis factorial confirmatorio mostró un buen ajuste de la estructura propuesta de cuatro factores (reforzamiento positivo, involucramiento parental, inconsistencia disciplinaria y prácticas punitivas). Inconsistencia disciplinaria, prácticas punitivas y reforzamiento positivo mostraron relación con la presencia de conductas desadaptativas en niños y niñas. Los resultados avalan la utilidad de la forma breve del Cuestionario de Alabama para su uso en la evaluación de prácticas parentales en familias de niños y niñas preescolares.
\end{abstract}

Palabras clave: prácticas parentales, preescolares, Cuestionario de Parentalidad de Alabama, propiedades psicométricas, análisis factorial confirmatorio

\begin{abstract}
The Alabama Parenting Questionnaire (APQ) is a widely used instrument to assess parenting practices. Recent studies show positive psychometric qualities and a robust factorial structure of a brief form. The aim of this study was to assess the psychometric properties of this brief form with parental figures of children from two to six years old in Chile. Participants were parental figures, mainly mothers, from a sample of 558 boys and girls from 2 to 6 years old. The confirmatory factor analysis showed a good adjustment for the factor (positive reinforcement, parental involvement, disciplinary inconsistency and punitive practices) proposed four-factor structure. Disciplinary inconsistency, punitive practice and positive reinforcement were related to disadaptative behavior in children. Overall, results support the use of Alabama's brief form in the assessment of parental practices in Chilean preschoolers' families.
\end{abstract}

Keywords: parenting practices, preschoolers, Alabama Parenting Questionnaire, psychometric properties, factorial confirmatory analysis

Este estudio fue financiado por el proyecto FONDECYT 11200716.

${ }^{1}$ Doctor en Psicología, Profesor Asociado, Dpto. de Psicología, Facultad de Ciencias Sociales, Universidad de Concepción, Barrio Universitario s/n, Concepción, Región del Bío Bío, Chile, Tel.: +56412204301. Correo: fecova@udec.cl

${ }^{2}$ Candidato a Doctor, Dpto. de Psicología, Facultad de Ciencias Sociales, Universidad de Concepción, Barrio Universitario s/n, Concepción, Región del Bío Bío, Chile. Tel.: +56412204301. Correo: cbustos@udec.cl

${ }^{3}$ Doctora en Psicología, Profesora Asociada, Dpto. de Psicología, Facultad de Ciencias Sociales, Universidad de Concepción, Barrio Universitario s/n, Concepción, Región del Bío Bío, Chile. Tel.: +56412204301. Correo:princon@udec.cl

${ }^{4}$ Doctora en Psicología, Profesora Asociada, Dpto. de Psicología, Facultad de Ciencias Sociales, Universidad de Concepción, Barrio Universitario s/n Concepción, Región del Bío Bío, Chile. Tel.: +56412204301. Correo: pgrandon@udec.cl

${ }^{5}$ Doctora en Psicología, Profesora Titular, Departamento de Psiquiatría y Salud Mental, Facultad de Medicina, Universidad de Concepción, Av. Juan Bosco s/n 3er piso, Concepción, Región del Bío Bío, Chile. Tel.: +56412312799. Correo: ssaldivi@udec.cl

${ }^{6}$ Doctora en Psicología, Profesora Asistente, Departamento de Psiquiatría y Salud Mental, Facultad de Medicina, Universidad de Concepción, Av. Juan Bosco s/n 3er piso, Concepción, Región del Bío Bío, Chile. Tel.: +56412312799. Correo: caroinostroza@udec.cl (autor para correspondencia)

Revista Iberoamericana de Diagnóstico y Evaluación - e Avaliação Psicológica. RIDEP · N51 Vol.2 · 33-42 2019

ISSN: 1135-3848 print /2183-6051online 


\section{Introducción}

Dada la relevancia de las prácticas parentales en el bienestar y desarrollo psicosocial de niños y niñas, existe necesidad de contar con herramientas para su evaluación (Galaz, Ayala, \& Coronado, 2009) tanto con propósitos clínicos como de investigación (Hawes \& Dadds, 2006). La herramienta empleada más habitualmente son los cuestionarios, ya sea para que las figuras parentales autoinformen de la frecuencia con que realizan determinados comportamientos con sus hijos e hijas, o para que estos últimos informen de los comportamientos que perciben que sus madres o padres tienen con ellos. Aunque los cuestionarios están sujetos a diversos sesgos y limitaciones, existen evidencias de la utilidad que pueden tener para algunos propósitos (Flores \& Herrera, 2014; Morsbach \& Printz, 2006).

Un instrumento de amplio uso y difusión internacional, traducido a más de 9 idiomas (Maguin, Nochajski, Dewit, \& Safyer, 2016), es el Cuestionario de Parentalidad de Alabama (CAP) (Shelton, Frick, \& Wootton, 1996). El CAP ha mostrado positivas propiedades psicométricas en diversos estudios (Badahdah \& Le, 2016; Esposito, Servera, Garcia-Banda et al., 2016), y es reconocido como uno de los mejores cuestionarios disponibles para el propósito de evaluar prácticas parentales específicas (Morsbach \& Prinz, 2006). El instrumento tiene una forma de autoinforme aplicable a las madres o padres y otra para ser respondida por los niños y niñas. Es apreciado por capturar diversas dimensiones relevantes de las prácticas parentales (Dadds, Maujean, \& Fraser, 2003). Su versión original, de 42 ítems, fue diseñada para evaluar 5 dimensiones de las prácticas parentales identificadas por distintos modelos teóricos y empíricos como factores de riesgo de comportamientos externalizados: 1. parentalidad positiva o prácticas positivas (referida al uso de reforzamiento positivo; para evitar confusiones, en este estudio se le denominará reforzamiento positivo); 2. involucramiento parental en las actividades del hijo/hija; 3. monitoreo y supervisión; 4. inconsistencia en las prácticas disciplinarias; y 5. prácticas punitivas (castigo físico). Algunos reactivos de dicha versión original están destinados a evaluar algunas prácticas parentales específicas no correspondientes a ninguna de las dimensiones señaladas.

Dada la utilidad que ha mostrado el CAP, ha existido interés en desarrollar formas breves de él. Un primer estudio con este fin (Elgar, Waschbusch, Dadds, \& Sigvaldason, 2007) propone una forma de 9 ítems; este estudio considera pertinente excluir las dimensiones de involucramiento y de prácticas punitivas. Un segundo estudio desarrolla una forma 15 ítems (Scott, Briskman, \& Dadds, 2011). En esta segunda investigación, se analizó una muestra clínica y comunitaria de 208 niños y niñas con edades de entre 9 y 17 años del Reino Unido; se seleccionaron los 3 ítems de cada una de las cinco dimensiones originales del CAP que habían mostrado ser más robustas a través de los diferentes estudios existentes, y se realizó un análisis factorial confirmatorio, que obtuvo un excelente ajuste (tanto en la forma de autoinforme para madres y padres como en la forma contestada por el niño o niña); se observó, asimismo, convergencia entre las distintas formas de medida y capacidad de discriminar entre niños y niñas con problemas de conducta.

$\mathrm{Ni}$ el CAP ni sus formas breves se desarrollaron para población preescolar. Clerkin y cols. (Clerkin, Marks, Policaro, \& Halperin, 2007) lo adaptaron a esta población. Realizaron un estudio con una muestra clínica y de comparación de niños y niñas entre 3 y 6 años, a quienes siguieron por un año. Excluyeron en función de su contenido los ítems no pertinentes para este grupo etario. Los resultados indicaron una adecuada consistencia interna y estabilidad temporal de los factores, así como sensibilidad al cambio al comparar al grupo clínico y control (Clerkin et al., 2007). De la Osa et al. (2014), en niños y niñas preescolares españoles, replicaron los hallazgos de Clerkin et al. (2007); como indicador de validez de criterio, observaron relaciones entre las medidas de inconsistencia disciplinaria y prácticas punitivas de esta forma adaptada del CAP para preescolares, con problemas y trastornos de conducta e impedimento funcional. Ambos estudios con preescolares mostraron que la dimensión monitoreo y supervisión del CAP original no era pertinente, en la forma como estaba formulada, en población preescolar, por lo que requiere ser excluida. 
A la fecha, no existen estudios de una forma breve del CAP en población preescolar. En el presente estudio se analiza una versión breve del CAP, en su forma de autoinforme para madres y padres, en población preescolar chilena. Específicamente, se analiza la bondad de ajuste de la forma breve generada por Scott et al. (2011). La única diferencia con esta forma es que se excluye el factor monitoreo y supervisión. Se hipotetiza que el modelo propuesto tendría una adecuada bondad de ajuste y que estas medidas breves de prácticas parentales mostrarían relación con la presencia de comportamientos desadaptativos en niños y niñas, en particular, de tipo externalizado.

\section{Método}

\section{Participantes}

Para el estudio se fusionaron dos muestras con las que se ha trabajado en investigaciones previas, obteniéndose un total de 550 participantes, 284 niños (52\%) y 266 (48\%) niñas. El rango de edad de los participantes fue de 2 a 6 años recién cumplidos ( $\mathrm{M}=3.4, \mathrm{DS}=.93)$.

Los y las participantes fueron obtenidos a través de muestreo intencionado en los establecimientos seleccionados. Un $45 \%$ corresponde a la medida pre-test obtenida de familias participantes, ya sea en calidad de grupo experimental o control, de un ensayo clínico randomizado de un programa de entrenamiento parental; otro 55\% son familias evaluadas en el contexto de un estudio solo transversal.

Esta muestra es diversa socioeconómicamente y fue obtenida a través de establecimientos educacionales de las ciudades de la provincia de Concepción, Chile. En Chile el tipo de establecimiento educacional a que se asiste es un buen indicador indirecto del nivel socioeconómico (NSE): 190 participantes (34.54\%) asisten a instituciones educativas privadas pagadas (NSE alto y medio alto) y 360 (65.45\%), a instituciones educativas públicas o fundaciones educacionales focalizadas en población económicamente vulnerable (NSE bajo). Este último grupo socioeconómico está algo sobre-representado respecto de la distribución socio-económica de Chile. En la Tabla 1 se puede observar la distribución de participantes por edad y NSE.

Con respecto a la estructura familiar, 387 participantes $(70.4 \%)$ viven con sus dos padres, 150 participantes $(27.3 \%)$ viven ya sea con el padre o la madre y los 13 restantes (2.3\%) viven con otras personas. La mediana de edad de las madres se encuentra entre los 30 y 35 años, y la mediana de educación se ubica en la educación media (secundaria) completa. Los instrumentos fueron a aplicados a las figuras parentales de los niños y niñas (madres, en su mayoría, 479, 87.1\% del total) y a sus educadoras (solo el cuestionario C-TRF). Se utilizó una submuestra de 83 participantes para estimar la confiabilidad testretest del instrumento, con una segunda evaluación a los 6 meses después. Este grupo está compuesto por participantes de NSE bajo, con madres de menor edad ( $p=.002$, Med=26-30 v/s Med=30-35) y educación $(p<0.001$, Med=Media Completa v/s Med=Educación Superior) con respecto al resto de la muestra.

\section{Instrumentos}

CAP Cuestionario de Parentalidad de Alabama (Shelton et al., 1996). Para este estudio, se seleccionaron los mismos ítems de la forma breve desarrollada por Scott et al. (2011). Esta forma considera tres ítems por cada factor, en formato de respuesta tipo Likert de 5 opciones (desde $1=$ Nunca a $5=$ Siempre), por lo que son 12 ítems en total (considerando que el factor monitoreo y supervisión fue excluido).

En esta investigación se utilizó la forma aplicada a la madre o al padre. Se empleó la

Tabla 1. Distribución de los participantes por nivel socio-económico (NSE) y edad

\begin{tabular}{ccccccc}
\hline \multirow{2}{*}{ Edad } & \multicolumn{2}{c}{ NSE Bajo } & \multicolumn{2}{c}{ NSE Alto/Medio } & \multicolumn{2}{c}{ Total } \\
\cline { 2 - 7 } & $\mathrm{N}$ & $\%$ & $\mathrm{~N}$ & $\%$ & $\mathrm{~N}$ & $\%$ \\
\hline 2 años & 32 & 5.8 & 49 & 8.9 & 81 & 14.7 \\
3 años & 189 & 34.4 & 49 & 8.9 & 238 & 43.3 \\
4 años & 97 & 17.6 & 49 & 8.9 & 146 & 26.5 \\
5 años & 40 & 7.3 & 43 & 7.8 & 83 & 15.1 \\
6 años & 2 & .4 & 0 & 0 & 2 & .4 \\
Total & 360 & 65.5 & 190 & 34.5 & 550 & $100 \%$ \\
Revista Iberoamericana de Diagnóstico y Evaluación-e Avaliação Psicológica. RIDEP $\cdot \mathrm{N}^{\circ} 51 \cdot$ Vol.2 $\cdot 33-42 \cdot 2019$ & &
\end{tabular}


traducción al castellano realizada por Roberts (2009) para su uso en México. Se hizo una aplicación piloto con 10 familias para evaluar la comprensibilidad de los ítems. Los resultados de este pilotaje indicaron que era conveniente reemplazar la palabra "halagar" por "felicitar". Las consistencias internas de los factores del Cuestionario de Alabama para la muestra completa fueron $\alpha=.70$ para el factor Refuerzo Positivo (p.e., "felicita a su hijo/a cuando hace algo bien"), $\alpha=.54$ para el factor Involucramiento Parental (p.e., juega o hace otras cosas divertidas con su hijo/a), $\alpha=.80$ para el factor Inconsistencia Parental (p.e., "amenaza a su hijo con que lo va a castigar, pero no lo castiga") y $\alpha=.45$ para Prácticas Punitivas (p.e., "le grita a su hijo cuando él o ella ha hecho algo malo o incorrecto").

Child Behavior Checklist 1/1/2 (CBCL 1/2) y Teacher Report Form, (C-TRF) (Achenbach \& Rescorla, 2000). CBCL $1 \frac{1 / 2}{2}$ y C-TRF son instrumentos de 100 ítems cada uno con tres opciones de respuesta tipo Likert (de $0=$ "No es cierto" a 2="Muy cierto o cierto a menudo"), que evalúan la presencia de conductas desadaptativas en niños y niñas preescolares (p.e., "Dolores o malestares"). El CBCL $1 \frac{1}{2}$ es respondido por la madre, padre o figura parental y el C-TRF, por personal educativo a cargo del niño o niña que tenga contacto frecuente con él. Están traducidos a diversos idiomas, incluyendo el castellano. Sus propiedades psicométricas han sido corroboradas en diversas investigaciones, incluyendo estudios en Chile (Lecannelier et al., 2014). Se emplearon las escalas globales de comportamientos internalizados y externalizados. La consistencia interna de la escala de internalización y externalización del CBCL $1 \frac{1}{2}$ fue de $\alpha=.86$ y $\alpha=.87$, y en del C-TRF de $\alpha=.86$ y $\alpha=.95$.

\section{Procedimiento}

El estudio fue aprobado por el Comité de Ética de la Universidad de Concepción, y consideró los principios de la Declaración de Helsinki.

El C-TRF fue aplicado como autoinforme a las educadoras de los niños y niñas. El CAP y CBCL lo aplicó un entrevistador que leía junto a la figura parental las preguntas, excepto en familias de sectores medios y altos donde no existían dudas del entrenamiento lector de quien contestaba las escalas. El C-TRF fue respondido directamente por las educadoras.

\section{Análisis Estadísticos}

Se emplearon los softwares Mplus 6 para el análisis factorial exploratorio y R 3.1.2 para el resto de los análisis. Se realizó un análisis factorial confirmatorio del modelo de cuatro factores relacionados, usando una matriz de correlaciones policóricas y estimación WLSMV, con parametrización theta. Como indicadores de ajuste se consideraron chi-cuadrado, chi-cuadrado normalizado, CFI, TLI y RMSEA. Se considera que un modelo se ajusta de manera adecuada si tanto CFI como TLI son superiores a $.95 \mathrm{y}$ RMSEA es menor a .05 (Williams \& O'Boyle, 2011). Se realizó asimismo análisis de invarianza métrica por sexo y por nivel socioeconómico adaptada a datos categóricos. Se consideraron como criterios tanto las diferencias de chicuadrado entre los distintos niveles de invarianza ( $p>.05)$ como la diferencia de CFI (<.01), menos sensible al tamaño de la muestra que el chicuadrado (Bollen, 1989; Byrne, Shavelson, \& Muthen, 1989; Cheung \& Rensvold, 2002).

Se realizó un análisis de Mokken (Mokken, 1971) del factor de prácticas punitivas, un tipo de análisis no paramétrico de la teoría de respuesta al ítem. Se utilizó este análisis para confirmar que la suma total en la escala respondiese a un nivel de medición ordinal, pese a las dificultades que mostró su análisis de consistencia interna realizado desde la teoría clásica de medición.

Como indicador de validez de criterio se realizaron regresiones lineales múltiples de la relación entre los puntajes del CAP y los puntajes brutos de las escalas de externalización e internalización del CBCL 11/2 y C-TRF. Usando los criterios de Cohen (1992), se puede señalar que un $\mathrm{R}^{2}=.02$ se considera un tamaño del efecto pequeño, $\mathrm{R}^{2}=.13$ un tamaño del efecto moderado y $\mathrm{R}^{2}=.26$ un tamaño del efecto grande.

\section{Resultados}

El modelo de cuatro factores (Figura 1), si bien no mostró un ajuste absoluto a los datos, $\chi 2(48)=100.697, \quad p<.001, \quad$ obtuvo un ajuste adecuado en todos los indicadores considerados: 


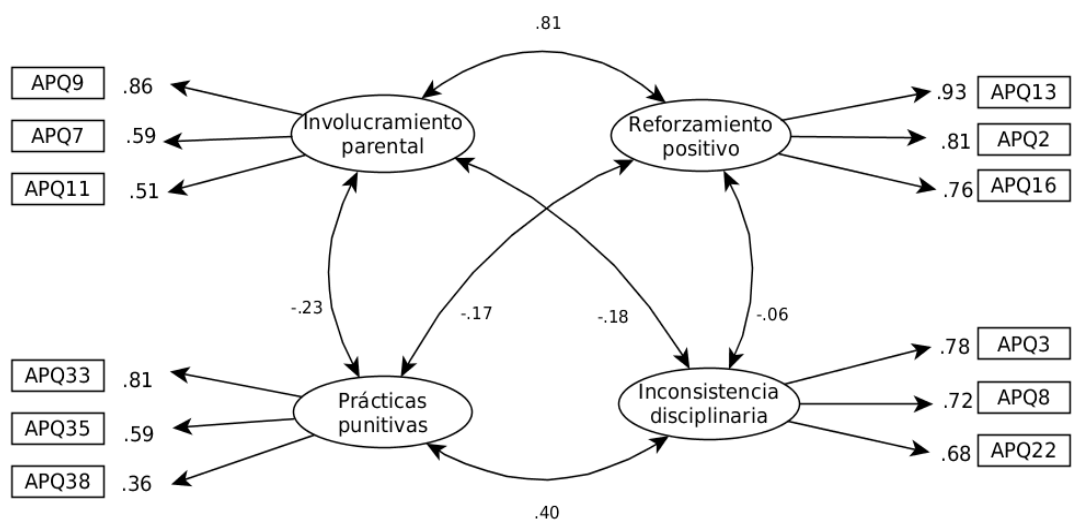

Figura 1. Estructura factorial del CAP forma breve con cargas factoriales y correlaciones entre factores

Tabla 2. Índices de ajuste del análisis de invarianza del CAP forma breve por sexo

\begin{tabular}{|c|c|c|c|c|c|c|c|c|c|c|c|c|}
\hline & $\chi^{2}$ & $\mathrm{gl}$ & Valor $p$ & $\chi^{2} / g 1$ & $\Delta \chi^{2}$ & $\mathrm{gl}$ & Valor $p$ & CFI & $\Delta \mathrm{CFI}$ & TLI & RMSEA & Valor $p$ \\
\hline \multicolumn{13}{|l|}{$\begin{array}{l}\text { Modelos } \\
\text { independientes }\end{array}$} \\
\hline Femenino & 69.38 & 48 & $<.020$ & 1.45 & & & & .971 & & .960 & .041 & .751 \\
\hline Masculino & 91.21 & 48 & $<.001$ & 1.90 & & & & .955 & & .938 & .056 & .263 \\
\hline $\begin{array}{l}\text { Invarianza } \\
\text { configuracional }\end{array}$ & 161.39 & 96 & $<.001$ & 1.68 & & & & .961 & & .947 & .050 & .495 \\
\hline Débil & 164.57 & 104 & $<.001$ & 1.58 & 6.89 & 8 & .549 & .964 & .003 & .954 & .046 & .678 \\
\hline Fuerte & 183.50 & 125 & $<.001$ & 1.47 & 17.11 & 20 & .646 & .965 & .001 & .962 & .042 & .857 \\
\hline Estricta & 194.66 & 136 & $<.001$ & 1.43 & 12.63 & 12 & .396 & .965 & .000 & .966 & .040 & .920 \\
\hline
\end{tabular}

$\chi 2 \quad$ normalizado $=1.86 ; \quad \mathrm{CFI}=.969 ; \quad \mathrm{TLI}=.958$; RMSEA $=.045, \quad p=.751$. Todas las cargas factoriales fueron superiores a .51 , excepto la del ítem 38 del factor de prácticas punitivas.

Al realizar el análisis de cada sexo por separado, el modelo ajusta muy bien en las mujeres, con todos los indicadores dentro de lo esperado; en el caso de los hombres, el TLI=.938 es ligeramente bajo, y el RMSEA=.056 $p=.263$ ligeramente alto, pero no significativo. $\mathrm{Al}$ analizar los índices de modificación, no se encontró ninguno que fuese mayor a 20 , y que fuese factible de implementar sin alterar el modelo teórico, por lo que se decidió continuar con el análisis de invarianza sin realizar búsqueda de especificación. En la Tabla 2 se puede observar que se presenta invarianza métrica estricta por sexo, tanto al analizar las diferencias en ChiCuadrado entre los distintos modelos sucesivos de invarianza, así como las diferencias en CFI, que siempre son inferiores a .01.

Al realizar el análisis por separado de los niveles socioeconómico NSE medio/alto con NSE bajo (Tabla 3), el modelo del nivel bajo se ajustó bien a los datos; el nivel medio/alto no pudo ser estimado, por dificultades para estimar la varianza del factor prácticas punitivas. La carga factorial en este nivel socioeconómico entre la conducta "pegar con una correa" (ítem 38) y el factor de prácticas punitivas era casi 0 , lo que se puede deber a que la gran mayoría de los casos no realiza esta conducta. Al fijar el coeficiente de ese ítem a 0 , se logró estimar un modelo con buen ajuste.

La invarianza métrica por nivel socioeconómico se mantiene sólo a nivel configuracional y en nivel débil en sus cargas factoriales. Al modelar la invarianza fuerte, tanto la diferencia de Chi-cuadrado resulta significativa como el criterio práctico de diferencia de $\mathrm{CFI}$ (.012), que resulta ser mayor a .01. Realizando una búsqueda de especificación mediante los índices de modificación, se encontró que al liberar la relación entre los residuos de los ítems 9 y 11, pertenecientes a involucramiento parental, si bien se mantiene la diferencia significativa de Chi cuadrado, se logra disminuir la diferencia de CFI a .005 , lo que indica invarianza fuerte en términos prácticos. Finalmente, se observa que no se logra obtener invarianza estricta, ya que la diferencia de Chi-Cuadrado resulta significativa por un amplio margen, así como también la diferencia de CFI. 
Tabla 3. Índices de ajuste del análisis de invarianza del CAP forma breve por nivel socioeconómico (NSE)

\begin{tabular}{lcccccccccccc}
\hline \multicolumn{1}{l}{} & $\chi^{2}$ & $\mathrm{gl}$ & Valor $p$ & $\chi^{2} / \mathrm{gl}$ & $\Delta \chi^{2}$ & $\mathrm{gl}$ & Valor $p$ & CFI & $\Delta$ CFI & TLI & RMSEA Valor $p$ \\
\hline $\begin{array}{l}\text { Modelos } \\
\text { independientes }\end{array}$ & & & & & & & & & & & & \\
Bajo & 81.314 & 48 & .002 & 1.69 & & & & & & & & \\
Medio/Alto & 75.018 & 49 & .010 & 1.53 & & & & .968 & .956 & .044 & .715 \\
Invarianza & 155.293 & 97 & $<.001$ & 1.60 & & & & .967 & .953 & .053 & .399 \\
configuracional & & & & & & & & .967 & & .955 & .047 & .639 \\
Débil & 168.192 & 104 & $<.001$ & 1.62 & 14.00 & 7 & .051 & .964 & .003 & .954 & .047 & .616 \\
Fuerte & 208.032 & 124 & $<.001$ & 1.68 & 50.96 & 20 & $<.001$ & .952 & .012 & .949 & .050 & .506 \\
Fuerte2 $2^{\mathrm{b}}$ & 195.374 & 123 & $<.001$ & 1.59 & 31.68 & 19 & .034 & .959 & .005 & .956 & .046 & .684 \\
Estricta $^{2}$ & 268.467 & 135 & $<.001$ & 1.99 & 78.29 & 12 & $<.001$ & .924 & .035 & .926 & .060 & .060 \\
\hline
\end{tabular}

Nota. ${ }^{a}$ Se fija a 0 la carga del ítem 38 al factor de Prácticas Punitivas en el grupo Medio/Alto. ${ }^{b}$ Se deja libre la relación entre los residuos de los ítems 9 y 11 en el grupo Medio/Alto

Tabla 4. Descriptivos, consistencia interna y correlación test-retest de los factores del CAP forma breve

\begin{tabular}{|c|c|c|c|c|c|}
\hline Factor & M & $\mathrm{DE}$ & alpha & $\begin{array}{c}\text { omega } \\
\text { total }\end{array}$ & $\begin{array}{l}\text { test- } \\
\text { retest }^{\mathrm{a}}\end{array}$ \\
\hline $\begin{array}{l}\text { Reforzamiento } \\
\text { positivo }\end{array}$ & 3.78 & .41 & .70 & .82 & .58 \\
\hline $\begin{array}{l}\text { Involucramiento } \\
\text { parental }\end{array}$ & 3.51 & .53 & .43 & .54 & .64 \\
\hline $\begin{array}{l}\text { Inconsistencia } \\
\text { disciplinaria }\end{array}$ & 1.53 & .99 & .72 & .80 & .58 \\
\hline $\begin{array}{l}\text { Prácticas } \\
\text { punitivas }\end{array}$ & 0.46 & .42 & .34 & .45 & .48 \\
\hline
\end{tabular}

Nota. $\mathrm{n}=550 .{ }^{\mathrm{a}} \mathrm{n}=83$

Los factores mostraron correlaciones entre sí en las direcciones esperadas (Figura 1), observándose una fuerte relación entre reforzamiento positivo e involucramiento parental.

Con un subconjunto de 83 participantes que recibieron una segunda evaluación 6 meses después, se estimó la confiabilidad test-retest de los factores. Si comparamos esta submuestra con la muestra total, en las escalas de comportamiento disruptivo CBCL, se observan mayores valores en el grupo en que se calculó test-retest, tanto en comportamiento internalizado $(p=.02, \mathrm{M}=.35$ vs $\mathrm{M}=.42$ ) como externalizado ( $p=.003, \mathrm{M}=.69$ vs $\mathrm{M}=.81$ ). No se observan diferencias significativas en estructura familiar $(p=.32)$, media de edad de los niño/as $(p=.79)$, sexo de los niño/as ( $p=.08)$, ni en los factores del CAP en la medición pre-test.

En la Tabla 4 se informan los valores descriptivos de los puntajes de cada factor, su consistencia interna y la confiabilidad test-retest, la que osciló entre .48 (prácticas punitivas) y .64 (involucramiento parental).
En la escala de prácticas punitivas se observó un patrón análogo al de las escalas Guttman, donde la presencia de prácticas de mayor gravedad predecía con alta probabilidad la presencia de las conductas de menos gravedad. Sin embargo, la muy baja frecuencia de estas conductas implica que la correlación máxima que puede existir entre ellas se encuentra limitada y, por tanto, existe un límite artificial a la consistencia interna medida desde la teoría clásica. Para estudiar este patrón, se realizó un análisis de Mokken (ver Tabla 5), que corresponde a una versión probabilística de la escala de Guttman (Mokken, 1971). Considerando los supuestos de este tipo de análisis, se evaluó, en primer lugar, la monotonicidad, verificándose que a mayores sumas totales de la escala aumente la proporción de valores superiores en los ítems individuales, lo cual se cumplió para todos los ítems. En segundo lugar, se analizó el supuesto de orden invariante de los ítems, el cual verifica que cada ítem es consistentemente más difícil que otro, en todos los niveles de la suma total de ítems, lo cual también se cumplió.

El coeficiente HT, que mide en qué medida el patrón de la respuesta se asemeja a una escala Guttman fue de HT=.89, lo cual indica que una respuesta de mayor intensidad en un ítem de mayor dificultad asegura una respuesta de mayor intensidad en los de menor dificultad. Todos los ítems superan un indicador de escalabilidad parcial de .2, por lo que, de acuerdo al análisis de Mokken, todos los ítems son útiles para medir la conducta punitiva, indicando así la confiabilidad de la escala. 
Tabla 5. Dificultad e índice de escalabilidad del factor prácticas punitivas

\begin{tabular}{lccc}
\hline Ittem & $\mathrm{M}$ & $\mathrm{DE}$ & $\mathrm{H}_{\mathrm{i}}$ \\
\hline 33 & 1.13 & .93 & .37 \\
35 & .17 & .49 & .37 \\
38 & .09 & .37 & .21 \\
\hline Nota. $\mathrm{H}_{\mathrm{i}}$ : Índice de escalabilidad & &
\end{tabular}

Nota. $\mathrm{H}_{\mathrm{i}}$ : Índice de escalabilidad

Tabla 6. Predicción del CBCL externalizado e internalizado a partir del CAP forma breve

\begin{tabular}{lcccc}
\hline & \multicolumn{2}{c}{ CBCL externalizado } & \multicolumn{2}{c}{ CBCL internalizado } \\
\cline { 2 - 5 } & $\mathrm{B}$ & $\mathrm{IC} 95 \%$ & $\mathrm{~B}$ & $\mathrm{IC} 95 \%$ \\
\hline Reforzamiento positivo & $-.086^{*}$ & {$[-.165,-.006]$} & .006 & {$[-.079, .092]$} \\
Involucramiento parental & .015 & {$[-.065, .095]$} & -.057 & {$[-.142, .029]$} \\
Inconsistencia disciplinaria & $.390^{* *}$ & {$[.315, .465]$} & $.324 * *$ & {$[.244, .404]$} \\
Prácticas punitivas & $.220^{* *}$ & {$[.145, .295]$} & $.110^{* *}$ & {$[.030, .191]$} \\
$\mathrm{R}^{2}$ & .250 & & .141 & \\
$\mathrm{~F}$ & $45.22^{* *}$ & & $22.24 * *$ & \\
\hline Nota. B: Coeficiente de regresión estandarizado. ${ }^{*} p<.05 * * p<.01$ & &
\end{tabular}

Para establecer las relaciones entre los factores y conductas desadaptativas externalizadas e internalizadas en los niños y niñas se realizaron regresiones múltiples. Respecto de comportamientos externalizados informados por la figura parental, reforzamiento positivo, inconsistencia disciplinaria y prácticas punitivas mostraron ser predictores significativos (Tabla 6). Respecto de comportamientos internalizados informados por la figura parental, solo los dos últimos fueron predictores significativos (Tabla 6). Al analizar las diferencias en $\mathrm{R}^{2}$ entre la predicción de comportamientos internalizados y externalizados, se puede observar una diferencia de .109, indicando un mayor poder predictivo sobre externalización. Al utilizar 5000 muestras bootstrap y calcular la diferencia entre ambos coeficientes de determinación, se puede observar que el intervalo de confianza al 95\% IC $=[.047$, .166] no incluye el 0 , por lo que se puede concluir que esta diferencia es estadísticamente significativa.

El análisis de regresión sobre las escalas informadas por las educadoras no mostró resultados significativos ni para la escala externalizada, $\mathrm{F}(4.554)=2.119, p=.07$ ni para la escala internalizada, $\mathrm{F}(4.554)=1.145, p=.33$.

\section{Discusión}

Scott et al. (2011), en familias de niños y niñas ingleses de 9 a 17 años, obtuvieron resultados psicométricos positivos de una forma breve del CAP de 15 ítems. En este estudio se analizó esta forma breve en niños y niñas chilenos de 2 a 6 años. Se excluyeron a priori los ítems del factor supervisión y monitoreo, dado que se ha observado que los ítems del CAP para este factor no son pertinentes para población preescolar (Clerkin et al., 2007). Se configuró así una forma breve del CAP de 12 ítems.

El modelo de 4 factores de prácticas parentales mostró un ajuste satisfactorio. Los resultados son análogos a los de Scott et al. (2011) y validan la distinción entre las cuatro dimensiones de prácticas parentales consideradas, dos positivas (reforzamiento positivo e involucramiento parental) y dos negativas (inconsistencia disciplinaria y prácticas punitivas). Los factores de prácticas positivas y negativas se relacionan entre sí en la forma esperada. La relación entre reforzamiento e involucramiento es fuerte, lo que explica que en algunos estudios hayan sido tratados como un solo factor (Molinuevo, Pardo, \& Torrubia, 2011).

El modelo mostró ser estable en niños y niñas y en nivel socioeconómico bajo y medio/alto. Sin embargo, la consistencia interna de cada factor es reducida. En parte ello se explica por tratarse de factores de solo tres ítems. Ello podría generar dudas respecto de la confiabilidad del instrumento. Sin embargo, las correlaciones test- 
retest son adecuadas.

Como es habitual en estudios del CAP, el factor de prácticas punitivas presentó una consistencia muy baja (de la Osa, Granero, Penelo, Domenech, \& Ezpeleta, 2014; Elgar et al., 2007). Este factor considera algunos ítems que corresponden a conductas de baja presencia, pero de gran significado clínico ("golpea a su hijo/a con una correa..."). Dada la relevancia clínica de este factor, se realizó con él un tipo de análisis particular, sugerido por Mokken (1971). Este análisis mostró un ajuste adecuado $(\mathrm{HT}=.89)$, lo que implica que las respuestas que indican conductas punitivas más graves se relacionan con la presencia de respuestas de mayor intensidad en los ítems punitivos menos severos también. Hawes y Dadds (2006) encontraron que este factor fue el único que correlacionó con medidas observacionales de parentalidad hostil. Su relevancia clínica y teórica, su aceptable correlación test-retest y los adecuados indicadores de ajuste tanto del modelo de 4 factores como del propio factor, sugieren que sería pertinente conservarlo, así como los tres ítems que considera.

Las prácticas parentales evaluadas, en particular las negativas (inconsistencia parental y prácticas punitivas), mostraron relacionarse con mayores conductas desadaptativas percibidas por la figura parental, en particular, con las de tipo externalizado (en esta última, reforzamiento positivo también mostró poder predictivo). La menor capacidad predictiva de las dimensiones de reforzamiento positivo e involucramiento parental ya fue observada en el primer estudio publicado de uso del instrumento (Shelton et al., 1996), donde no mostraron capacidad de discriminar entre la muestra clínica y control; en general, se observa una menor relación de las prácticas parentales positivas con comportamientos desadaptativos (de la Osa et al., 2014; Molinuevo et al., 2011; Roberts, 2009; Scott et al., 2011). Debe tenerse presente que componentes relevantes de las prácticas parentales positivas como la calidez y la sensibilidad anticipatoria a la necesidades del niño o niña (Gardner, Dishion, Shaw, Burton, \& Supplee, 2007) no están consideradas adecuadamente en el CAP.

Dado que el CAP fue diseñado como instrumento para evaluar las dimensiones que más han mostrado influir en el desarrollo de problemas y trastornos de conducta, es esperable que muestre mayor relación con la presencia de comportamientos externalizados que internalizados. No se observaron, sin embargo, relaciones con los comportamientos externalizados e internalizados informados por las educadoras. La baja concordancia entre los informes de conductas desadaptativas de las figuras parentales y otros informantes está bien establecida (Achenbach \& Rescorla, 2000; Liu, Cheng, \& Leung, 2011). Dado que el CAP es respondido por las figuras parentales, es esperable una mayor relación de sus medidas con comportamientos desadaptativos informados también por ellas que respecto de los informados por las educadoras. Sin embargo, la nula relación observada requiere de investigaciones adicionales. Existen pocos estudios de la relación entre prácticas parentales medidas por el CAP y conductas desadaptativas percibidas por las educadoras en población preescolar (Molinuevo et al., 2011; Scott et al., 2011).

Una limitación de este estudio es que, al igual que Scott et al. (2011), se seleccionaron los ítems de la forma breve habiéndose aplicado la forma completa del instrumento a los participantes. Sería deseable establecer que la confiabilidad del instrumento no esté artificialmente inflada por efecto de esta manera de administración (Smith, Combs, \& Pearson, 2012). Otra limitación del presente estudio es que no consideró otras mediciones que permitieran obtener indicadores de validez convergente.

En síntesis, los resultados obtenidos de esta adaptación del CAP para la evaluación de prácticas parentales en población preescolar sugieren que el uso de esta forma breve del CAP con fines clínicos y de investigación en un país como Chile, y en población de distinto nivel socioeconómico, puede ser apropiada. Se trata de una herramienta sencilla y de rápida aplicación, que puede aportar información útil, pese a las limitaciones propias de una escala de autoinforme, para la evaluación de las prácticas parentales (Morsbach \& Prinz, 2006). 


\section{Referencias}

Achenbach \& Rescorla. (2000). Manual for the ASEBA preschool forms \& profiles: An integrated system of multi-informant assessment. Burlington, VT: ASEBA.

Badahdah, A. \& Le, K. T. (2016). Parenting young arab children: Psychometric properties of an adapted Arabic Brief Version of the Alabama Parenting Questionnaire. Child Psychiatry \& Human Development, 47(3), 486-493.

Bollen, K. A. (1989). A new incremental fit index for general structural equation models. Sociological Methods \& Research, 17(3), 303-316. doi:10.1177/0049124189017003004

Byrne, B. M., Shavelson, R. J., \& Muthen, B. (1989). Testing for the equivalence of factor covariance and mean structures - the issue of partial measurement invariance. Psychological Bulletin, 105(3), 456-466. doi:10.1037/0033-2909.105.3.456

Clerkin, S. M., Marks, D. J., Policaro, K. L., \& Halperin, J. M. (2007). Psychometric properties of the Alabama Parenting Questionnaire-Preschool Revision. Journal of Clinical Child and Adolescent Psychology, 36(1), 19-28. doi:10.1207/s15374424jccp3601_3

Cheung, G. W., \& Rensvold, R. B. (2002). Evaluating goodness-of-fit indexes for testing measurement invariance. Structural Equation Modeling, $\quad 9(2), \quad$ 233-255. doi:10.1207/S15328007sem0902_5

Cohen, J. (1992). A power primer. Psychological Bulletin, 112(1), 155-159. http://doi.org/10.1037/0033-2909.112.1.155

Dadds, M. R., Maujean, A., \& Fraser, J. A. (2003). Parenting and conduct problems in children: Australian data and psychometric properties of the Alabama Parenting Questionnaire. Australian Psychologist, 38(3), 238-241. doi:10.1080/00050060310001707267

de la Osa, N., Granero, R., Penelo, E., Domenech, J. M., \& Ezpeleta, L. (2014). Psychometric properties of the Alabama Parenting Questionnaire-Preschool Revision (APQ-Pr) in 3 year-old Spanish preschoolers. Journal of Child and Family Studies, 23(5), 776-784. doi:10.1007/s10826-013-9730-5
Elgar, F., Waschbusch, D., Dadds, M., \& Sigvaldason, N. (2007). Development and validation of a short form of the Alabama Parenting Questionnaire. Journal of Child and Family Studies, 16, 243-259. doi:10.1007/s10826-006-9082-5.

Esposito, A., Servera, M., Garcia-Banda, G. et al. (2016). Journal of Child and Family Studies, 16(25), 1208. doi:https://doi.org/10.1007/s10826-015-0291-7

Flores, J. J. \& Herrera, L. M. F. (2014). Design and psychometric validation of the Harsh Discipline Practice List. Revista Iberoamericana de Diagnóstico y Evaluación - e Avaliação Psicológica, 2(38), 137-153.

Galaz, M. M. F., Ayala, M. D. C., \& Coronado, E. A. G. (2009). Development and validation of the children rearing practices perception scale in a mexican sample. Revista Iberoamericana de Diagnóstico y Evaluación - e Avaliação Psicológica, 2(28), 45-66.

Gardner, F., Dishion, T. J., Shaw, D. S., Burton, J., \& Supplee, L. (2007). Randomized prevention trial for early conduct problems: Effects on proactive parenting and links to toddler disruptive behavior. Journal of Family Psychology, 21(3), 398-406. doi:10.1037/0893-3200.21.3.398

Gross, T. J., Fleming, C. B., Mason, W. A., \& Haggerty, K. P. (2017). Alabama parenting questionnaire-9: Longitudinal measurement invariance across parents and youth during the transition to high school. Assessment, 24(5), 646-659.

Hawes, D., \& Dadds, M. (2006). Assessing parenting practices through parent-report and direct observation during parent-training. Journal of Child and Family Studies, 15(5), 556-568. doi:10.1007/s10826-006-9082-5

Lecannelier, F., Ewert, J. C. P., Groissman, S., Gallardo, D., Bardet, A. M., Bascunan, A., \& Rodriguez, J. (2014). Validation of Child Behavior Inventory for children ages $11 / 2$ to 5 years (CBCL 1 1/2-5) at the Santiago de Chile City. Universitas Psychologica, 13(2), 491-500.

Liu, J. H., Cheng, H., \& Leung, P. W. L. (2011). The application of the Preschool Child Behavior Checklist and the Caregiver-Teacher Report Form to Mainland Chinese children: 
Syndrome structure, gender differences, country effects, and inter-informant agreement. Journal of Abnormal Child Psychology, 39(2), 251-264. doi: $10.1007 /$ s10802-010-9452-8

Maguin, E., Nochajski, T., Dewit, D., \& Safyer, A. (2016). Examing the Validity of the Adapted Alabama Parenting Questionnaire Parent Global Report Version. Psychological Assessment, 28(5), 613-625. doi:http://doi.org/10.1037/pas0000214

Mokken, R. J. (1971). A theory and procedure of scale analysis with applications in political research. The Hague: Mouton.

Molinuevo, B., Pardo, Y., \& Torrubia, R. (2011). Psychometric analysis of the Catalan version of the Alabama Parenting Questionnaire (APQ) in a Community Sample. Spanish Journal of Psychology, 14(2), 944-955. doi:10.5209/rev_SJOP.2011.v14.n2.40

Morsbach, S. K., \& Prinz, R. J. (2006). Understanding and improving the validity of self-report of parenting. Clinical Child and Family Psychology Review, 9(1), 1-21. doi:10.1007/s10567-006-0001-5

Roberts, C. (2009). Doctoral Thesis. Parenting practices and child bevavior in Mexico: A validation study of the Alabama Parenting Questionnaire. University of Minnesota.

Scott, S., Briskman, J., \& Dadds, M. R. (2011). Measuring parenting in community and public health research using brief child and parent reports. Journal of Child and Family Studies, 20(3), 343-352. doi:10.1007/s10826-010-9398-z

Shelton, K. K., Frick, P. J., \& Wootton, J. (1996). Assessment of parenting practices in families of elementary school-age children. Journal of Clinical Child Psychology, 25(3), 317-329. doi:10.1207/s15374424jccp2503_8

Smith, G. T., Combs, J. L., \& Pearson, C. M. (2012). In H. Cooper; P.M. Camic; D. L. Long; A. T. Panter; D. Rindskopf y K. J. Sher (Eds). APA handbook of research methods in psychology, Vol 1: Foundations, planning, measures, and psychometrics (pp. 395-409). Washington, DC, US: American Psychological Association, xliv, 744. doi:http://dx.doi.org/10.1037/13619-021

Williams, L. J., \& O’Boyle, E. (2011). The myth of global fit indices and alternatives for assessing latent variable relations. Organizational Research Methods, 14(2), 350369. doi: http://doi.org/10.1177/1094428110391472 